\title{
Antiresonant hollow core fiber with seven nested capillaries
}

Antonio-Lopez, Jose E.; Habib, Selim; Van Newkirk, Amy; Lopez-Galmiche, Gisela; Eznaveh, Zeinab S.; Alvarado-Zacarias, Juan C.; Bang, Ole; Bache, Morten; Schulzgen, Axel; Correa, Rodrigo Amezcua

\section{Published in:}

Proceedings of 2016 IEEE Photonics Conference

Link to article, DOI:

10.1109/IPCon.2016.7831157

Publication date:

2016

Document Version

Peer reviewed version

Link back to DTU Orbit

Citation (APA):

Antonio-Lopez, J. E., Habib, S., Van Newkirk, A., Lopez-Galmiche, G., Eznaveh, Z. S., Alvarado-Zacarias, J. C., Bang, O., Bache, M., Schulzgen, A., \& Correa, R. A. (2016). Antiresonant hollow core fiber with seven nested capillaries. In Proceedings of 2016 IEEE Photonics Conference (pp. 402-403). IEEE. https://doi.org/10.1109/IPCon.2016.7831157

\section{General rights}

Copyright and moral rights for the publications made accessible in the public portal are retained by the authors and/or other copyright owners and it is a condition of accessing publications that users recognise and abide by the legal requirements associated with these rights.

- Users may download and print one copy of any publication from the public portal for the purpose of private study or research.

- You may not further distribute the material or use it for any profit-making activity or commercial gain

- You may freely distribute the URL identifying the publication in the public portal 


\title{
Antiresonant Hollow Core Fiber with Seven Nested Capillaries
}

\author{
Jose E. Antonio-Lopez, ${ }^{1, *}$ Selim Habib ${ }^{1,2}$ Amy Van Newkirk ${ }^{1}$ Gisela Lopez-Galmiche, ${ }^{1}$ Zeinab S. \\ Eznaveh, ${ }^{1}$ Juan C. Alvarado-Zacarias, ${ }^{1}$ Ole Bang, ${ }^{2}$ Morten Bache, ${ }^{2}$ Axel Schülzgen, ${ }^{1}$ and Rodrigo \\ Amezcua Correa ${ }^{1}$ \\ ${ }^{I}$ CREOL, The College of Optics and Photonics, University of Central Florida, Orlando, Florida 32816, USA \\ ${ }^{2}$ DTU Fotonik, Department of Photonics Engineering, Technical University of Denmark, 2800 Kgs Lyngby, Denmark
} *iealopez@creol.ucf.edu

\begin{abstract}
We report an antiresonant hollow core fiber formed of 7 non-touching capillaries with inner tubes. The fiber has a core diameter of $\sim 33 \mu \mathrm{m}$ and a core wall of $\sim 780 \mathrm{~nm}$ of thickness. We demonstrate robust single mode operation at $1064 \mathrm{~nm}$ and broad transmission bandwidth. OCIS codes: (060.2330) Fiber optics communication; (060.2340) Fiber optics components
\end{abstract}

\section{Introduction}

Hollow-core fibers (HCFs) are a versatile fiber technology platform for numerous applications [1,2]. Since the early days of HCF research, it was identified that the core boundary strongly impacts the guiding properties of the fiber and its accurate control is required in order to minimize propagation losses [3]. In the past few years, rapid progress has been made in developing low-loss and bendable HCFs comprising an antiresonant, negative curvature core boundary. In antiresonant hollow-core fibers (AR-HCFs), the precisely designed core wall minimizes the optical intensity in the glass structure and reduces losses [2, 4-8]. The first demonstration of an AR-HCF was based on a Kagome structure [3]. This fiber design achieved relatively low transmission loss and broad-band guidance due to the low coupling between their core and cladding modes. Subsequently, various designs of AR-HCF based on a negative curvature core wall with improved optical properties were demonstrated [4-8]. Recently, non-touching capillaries core wall fibers were demonstrated as an attempt to further reduce the transmission losses and at the same time, broaden the transmission window [8]. In these fibers, the non-touching core wall maintains a constant thickness of the reflecting glass membrane, and thus suppressing the appearance of undesired resonances. However, the losses of these fibers are dominated by confinement loss. In order to overcome confinement loss, a novel fiber known as nested AR-HCF, utilizing secondary capillaries within each external capillary was proposed [8]. Numerical simulations have predicted that sophisticated nested AR-HCF can potentially achieve extremely low attenuation values [9]. However, in order to fabricate such complex AR-HCFs, accurate control of the core wall membrane and the secondary inner tubes structure is required.

Here, we report the fabrication and characterization of an open boundary 7-capillaries AR-HCF with nested tubes. The fiber was fabricated by the well-known stack and draw method using thin tubes to create the cladding. The measured optical properties of the fabricated fiber are in excellent agreement with numerical simulations performed using a scanning electron microscope (SEM) image of the actual fiber. The fiber was designed for single mode operation at $1064 \mathrm{~nm}$.
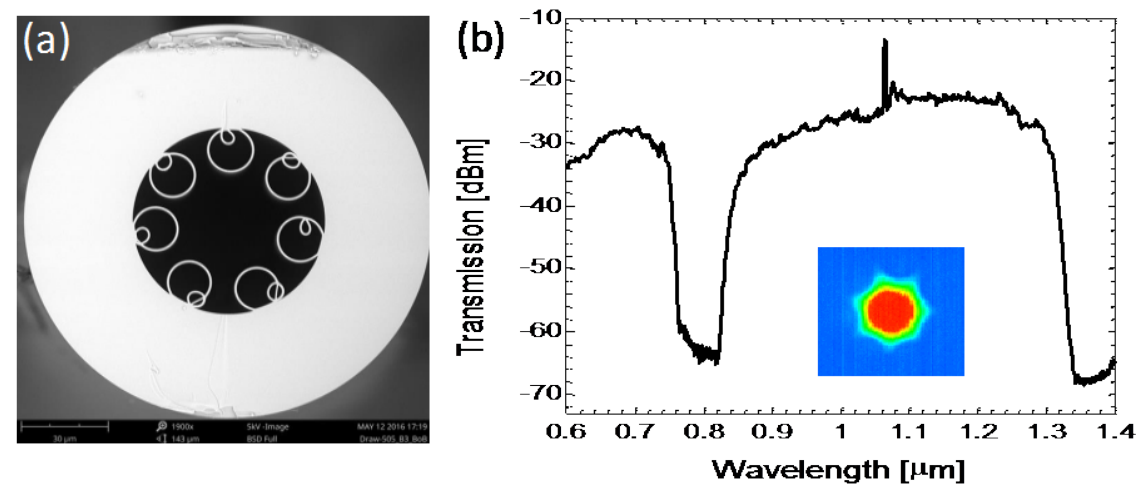

Fig. 1(a) SEM image of the fabricated 7-capillaries AR-HCF. (b) Measured transmission spectrum and near field mode profile obtained at $1064 \mathrm{~nm}$ 
Figure. 1(a) shows a SEM image of the fabricated AR-HCF. The fiber has an outer diameter of $124 \mu \mathrm{m}$, a core diameter of $33 \mu \mathrm{m}$ and a core wall thickness of $\sim 780 \mathrm{~nm} \pm 50 \mathrm{~nm}$. The wall thickness of the inner capillaries is $\sim 749$ $\mathrm{nm} \pm 50 \mathrm{~nm}$. The diameter of the larger cladding capillaries is $\sim 13.3 \mu \mathrm{m}$, while the diameter of the inner tubes is $\sim 3.3$ $\mu \mathrm{m}$. Although further fabrication improvements for controlling the shape and position of the inner capillaries are still required, we have solved important challenges related to the fabrication of double-capillary AR-HCF and demonstrate a complex fiber structure with a relatively thin core wall.

Figure. 1(b) shows the measured transmission spectrum for a $2 \mathrm{~m}$ long nested AR-HCF along with the near field mode profile obtained at $1064 \mathrm{~nm}$. The transmission spectrum was measured by using a broadband source (NKT photonics SuperK) via butt coupling with a single mode fiber at the input end of the AR-HCF. The transmission spectrum shows two resonant wavelengths $\sim 780 \mathrm{~nm}$ and $\sim 1380 \mathrm{~nm}$, which is in good agreement with the fiber's geometry. Using a cut-back measurement, a propagation loss of $\sim 2 \mathrm{~dB} / \mathrm{m}$ was measured at $1100 \mathrm{~nm}$. The mode profile image presented in Fig. 1(b), clearly shows that even for very short fiber lengths and a not optimized excitation, this fiber supports robust single operation.

In order to better understand the experimental results, we calculated the optical properties of the fabricated fiber using a FEM model (COMSOL). For these simulations, an accurate model of the actual fiber was obtained from the SEM image presented in Fig. 1(a) using image processing tools. Moreover, to precisely model the fiber confinement loss, we utilized a circular perfectly matched layer (PML) and both mesh and PML parameters were carefully optimized [10,11]. Fig. 2(a) presents the modeled structure and the calculated intensity profile of the fundamental mode at $1064 \mathrm{~nm}$. Fig. 2(b) shows remarkable agreement between the measured loss (black line) and the calculated confinement loss (red line) of the nested AR-HCF.
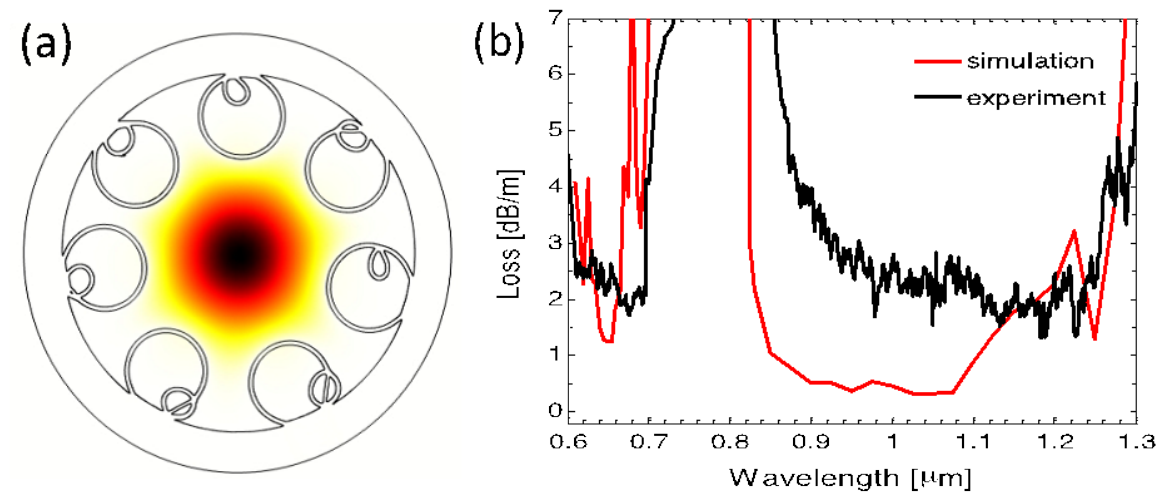

Fig. 2 (a) Cross-section of the simulated AR-HCF along with the calculated fundamental mode intensity profile at $1064 \mathrm{~nm}$. (b) Measured and simulated loss spectra.

In conclusion, we have fabricated a novel nested capillaries AR-HCF with a thin core wall boundary. The fiber's cladding consists of 7 non-touching capillaries with inner tubes that enhance the antiresonant reflection. The fiber has a core diameter of $\sim 33 \mu \mathrm{m}$ and a thin core wall. Experimental measurements and numerical simulations of the fabricated fiber are in excellent agreement. By further reducing the core wall thickness and improving the fiber structure we expect to significantly reduce losses and increase the transmission bandwidth.

This work was supported by HEL-JTO and ARO grant No. W911NF-12-1-0450 and AFOSR grant No. FA9550-15-10041.

\section{References}

1. J. C. Knight, "Photonic band gap guidance in optical fibers," Science, vol. 282, no. 5393. 1998.

2. M. Michieletto, et al., "Hollow-core fibers for high power pulse delivery," Opt. Express 24, 7103 (2016).

3. F. Benabid, et al., "Stimulated Raman scattering in hydrogen- 17. Filled hollow-core photonic crystal fiber.," Science 298, 399-402 (2002).

4. Y. Y. Wang, et al., "Low loss broadband transmission in hypocycloid-core Kagome hollow-core photonic crystal fiber.," Opt. Lett. 36, 669-71 (2011).

5. A. D. Pryamikov et al.,"Demonstration of a waveguide regime for a silica hollow-core microstructured optical fiber with a negative curvature of the core boundary in the spectral region > $3.5 \mu \mathrm{m}$.," Opt. Express 19, 1441-8 (2011).

6. Fei Yu, et al., "Low loss silica hollow core fibers for 3-4 $\mu \mathrm{m}$ spectral region," Opt. Express, vol. 20, no. 10. 2012.

7. A. N. Kolyadin, et al., "Light transmission in negative curvature hollow core fiber in extremely high material loss region.," Opt. Express 21, 9514-9 (2013).

8. W. Belardi et al., "Hollow antiresonant fibers with low bending loss.," Opt. Express 22, 10091-6 (2014).

9. $\quad$ F. Poletti, "Nested antiresonant nodeless hollow core fiber," Opt. Express, vol. 22, no. 20. 2014.

10. M. S. Habib, et al., "Low-loss hollow-core silica fibers with adjacent nested anti-resonant tubes," Opt. Express, vol. 23, no. 13.2015.

11. M. S. Habib, et al., "Low-loss single-mode hollow-core fiber with anisotropic anti-resonant elements," Opt. Express, vol. 24 , no. 8. 2016 